\title{
Research of cardiomyocyte precursors in adult rat heart
}

\author{
M. Bellafiore*, G. Sivverini, F. Cappello, S. David, \\ A. Palma, F. Farina, G. Zummo \\ Human Anatomy Section, Department of Experimental Medicine, University of Palermo, \\ Via del Vespro 129, 90127 Palermo, Italy
}

Received 7 April 2006; received in revised form 22 August 2006; accepted 23 August 2006

\begin{abstract}
Recent reports supported the existence of stem cells in adult hearts. However, phenotype and localization of these cells have not been completely described and it is unknown if cardiac regenerative potential differs from one subject to another. The aims of our work were to identify different populations of cardiac stem cells by the analysis of specific markers and to evaluate the expression variability of these markers in 12 adult rat hearts. The expression of CD9, taube nuss and nanog suggests the presence of stem cells from the earliest stages of embryogenesis in adult myocardium. Their different expression could be associated to the degree of stem cell differentiation. CD34 and c-Kit antibodies were used to detect stem cells committed to one or more specific tissue lineages and we found a strong immunoreactivity for CD34 exclusively in the endothelial cells and a low positivity for c-Kit in the interstitium and next to the vessels. Moreover, as c-Kit expression highly differed within all examined hearts, we suggest that cardiomyogenic potential is different among the various subjects. Undifferentiated cells with myogenic-committed phenotype expressing GATA-4 and nestin were found, respectively, in the interstitial and myocardial cells and in few interstitial cells. Therefore, the physiologic turn over of cardiomyocytes may occur in adult hearts as it has been shown in many others organs. The study of myogenic potential could be important to identify markers specific of stem cells in in vivo adult myocardium that may be used to purify these cells and evaluate their regenerative ability.
\end{abstract}

(c) 2006 Elsevier Ltd. All rights reserved.

Keywords: Cardiac resident stem cells; Rat myocardium; Cardiac progenitor markers

\section{Introduction}

Until recently, it was believed that adult stem cells were restricted in their ability to generate only the differentiated cell phenotypes of the host tissue. However, in the last years a number of publications have provided evidences for a greater plasticity of stem cell commitment and differentiation (Young and Black, 2004). For example, bone marrow cells, including isolated populations of hematopoietic stem cells have been reported to differentiate into muscle, neurons, liver, vascular, lung, intestine and kidney tissue (Yoder, 2004). In addition, it has been shown that stem cells obtained from adult bone marrow not only were able to differentiate into cardiomyocytes but also contributed to restore cardiac function when

\footnotetext{
* Corresponding author. Tel.: +39091655 3513; fax: +39091655 3580 . E-mail address: bellafiore@unipa.it (M. Bellafiore).
}

either injected directly into the injured adult heart (Tomita et al., 1999) or exposed to myocardium via circulation (Orlic et al., 2001). Two distinct types of stem cells are currently known to reside within adult bone marrow: hematopoietic and mesenchymal stem cells. Shintani et al. (2001) showed that the mobilization of $\mathrm{CD} 34^{+}$hematopoietic cells may be an important physiologic mechanism of cardiac tissue repair after heart failure. Other studies indicated that bone marrowderived mesenchymal stem cells when introduced into adult mouse or rat hearts integrated into myocardium and exhibited a myocyte phenotype (Tomita et al., 1999; Toma et al., 2002). Another study reported that liver-derived stem cells also exhibited myocardial potential when transplanted into the adult mouse heart (Malouf et al., 2001).

All these reports suggest that adult stem cells possess a broad phenotypic potential that enables them to differentiate into cell types that are different from their resident tissue. A 
possible explanation of this phenomenon could be the high plasticity of adult stem cells that, according to this model, possess great flexibility in their phenotypic potential, with their eventual cell fate controlled by extracellular signals (Orkin and Zon, 2002). However, recent studies showed a high degree of heterogeneity among stem cell populations within adult tissues (Young and Black, 2004). In particular, two categories of precursor cells that reside within the organs and tissues of postnatal animals have been identified as lineage-committed progenitors (unipotent, bipotent, tripotent or multipotent) and lineage-uncommitted pluripotent stem cells (Young and Black, 2004). The first are those cells that have left embryonic developmental continuum to become reserve precursor cells and that are involved in the maintenance and repair of tissues and organs throughout the life span of the individual. Each progenitor cell of a particular tissue lineage has a unique profile of cell surface cluster of differentiation markers; moreover, it is unresponsive to lineage induction agents that take actions outside their respective tissue lineages. Two subcategories of lineageuncommitted pluripotent stem cells showing many similar features have been isolated. However, only one subcategory expressed embryonic stem cell markers and maintained its ability to form cell types from all three primary germ layer lineages although derived from adults (Young and Black, 2004). Therefore, it has been proposed that every tissue within the body holds a complement of precursor cells denoting its embryological origin.

Embryonic stem cells (ESC), derived from the inner cell mass of pre-implantation embryos, have been recognized as the earliest stem cell population (Evans and Kaufman, 1981). This population can differentiate into all somatic tissue including germ cells. ESC highly express genes and candidates that can serve as markers to identify lineageuncommitted stem cells and may also contribute to assess the differentiation degree (Bhattacharya et al., 2004). For example, pluripotent ESC showed a high expression level of nanog, taube nuss and CD9, that are involved, respectively, in the propagation of ESC and maintaining their pluripotency (Chambers et al., 2003), in the survival of pluripotent cells of mouse early embryos (Voss et al., 2000) and in embryonic stem cell colony formation and cell viability increasement (Oka et al., 2002). In addition, it has been seen that CD9 protein binds c-Kit receptor in bone marrow stem cells (Oka et al., 2002) and it may have a critical role in etiology of testicular germ cell tumors $\left(\mathrm{TGCT}_{\mathrm{s}}\right)$, suggesting a link between enforced pluripotency and transformation (Giuliano et al., 2005).

Other recent studies reported about cardiac stem or progenitor cells from adult mammalian hearts (Oh et al., 2003; Messina et al., 2004), whose purification was based exclusively on the expression of a stem cell-related surface antigen as c-Kit. Indeed, c-Kit ${ }^{+}$and $\mathrm{Lin}^{-}$cells were found to be self-renewing, clonogenic and multipotent, exhibiting differentiation towards myogenic, smooth muscle, or endothelial cell lineage but failing to contract spontaneously. These cells showed an uncompletely differentiated phenotype, some of them being already committed to cardiac myogenic lineage, expressing GATA-4, NKx-2.5 and MEF-2 and they regenerated functional myocardium when injected into an ischemic heart (Beltrami et al., 2003). Other researchers discovered that rat normal heart contained resident nestin ${ }^{+}$neural-like stem cells and that nestin mRNA levels were increased in response to myocardial ischemia (El-Helou et al., 2005). Despite the existence of a so-called myocardial stem cell population has been suggested, it is not clear how this population contributes to the respective established cardiac cell types in the heart; at the same time, the phenotype of these cells has not been properly described. Also, at the moment, it is not known if the cardiac regenerative potential differs from one subject to another.

The aim of our work was to analyse, in adult rat hearts, the expression of surface molecules and intracellular markers specific for stem cells with lineage-uncommitted phenotype (CD9, taube nuss and nanog), stem cells committed to one or more specific tissue lineages (CD34 and c-Kit) and undifferentiated cells with myogenic-committed phenotype (GATA-4 and nestin). An interesting aspect of this study has been to evaluate the expression variability of the markers in a significant number of adult rat hearts.

\section{Materials and methods}

\subsection{Animal care}

Experiments were carried out on 12 Wistar male rats of 24-months-old. The investigation conforms to the Guide for the Care and Use of Laboratory Animals published by the US National Institutes of Health (NIH Publication No. 8523, revised 1996). Animals were anaesthetized with ether and the hearts were perfused with physiologic solution and formalin before they were excised.

\subsection{Immunohistochemistry}

In order to examine the localization and expression of pericentrin, nestin, c-Kit, GATA- 4 and CD34 proteins in adult rat heart, we performed immunostaining analyses on formalinfixed, paraffin-embedded sections using the specific primary antibodies. After incubation of the sections with serum-free protein block (DAKO, Carpinteria, USA) for $10 \mathrm{~min}$ at room temperature (RT), antibodies against pericentrin (BD Biosciences, USA), nestin (BD Bosciences), c-Kit (Stressgen Biotechnologies, Canada), GATA-4 (Santa Cruz Biotechnology, USA) and CD34 (BioGenex, USA) were added at the corresponding dilutions as shown in Table 1 . Nonimmune mouse serum was substituted for negative controls and anticonnexin-43 (BD Biosciences) was used like positive control. After incubation with biotinylated secondary antibody for 10 min at RT, AEC chromogen (DAKO) was used to develop the horseradish peroxidase (HRP)-streptavidin complex. The 
Table 1

Dilutions of primary antibodies for immunohistochemical analysis

\begin{tabular}{ll}
\hline Antibodies & Dilution \\
\hline Pericentrin & $1: 20$ \\
Nestin & $1: 20$ \\
C-Kit & $1: 100$ \\
GATA-4 & $1: 20$ \\
CD-34 & $1: 20$ \\
Connexin-43 & $1: 50$ \\
\hline
\end{tabular}

semi-quantitative expression of the examined proteins was assessed by two independent observers according to the cell percentage positive for each antibody on 10 high power fields $(\times 40)$ for each slide. This analysis was performed on a scale of three grades: $(+)<33 \%$ of positive elements; $(++) 33-66 \%$; $(+++)>66 \%$.

\subsection{Immunoblotting}

The expression levels of nestin, c-Kit and GATA-4 proteins were evaluated in adult rat heart by Western blot analysis. Biopsies from ventricle walls were homogenized in lysis buffer (50 mM Tris/HCl, $150 \mathrm{mM} \mathrm{NaCl}, 1 \% \mathrm{NP}-40,1 \mathrm{mM}$ EDTA, protease inhibitors, $\mathrm{pH}$ 7.5), containing protease inhibitors (Protease inhibitor cocktail set III, Calbiochem, Darmstadt, Germany) and centrifuged at 13,000 rpm for $10 \mathrm{~min}$ at $4{ }^{\circ} \mathrm{C}$. Supernatant fraction was collected and protein concentration was determined using a colorimetric assay (Bio-Rad, Philadelphia, USA). Protein samples of $50 \mu \mathrm{g}$ for each lane and a protein marker (Bio-Rad) were separated by SDS-PAGE and transferred to a nitrocellulose membrane. After $1 \mathrm{~h}$ incubation with a blocking buffer at RT, membranes were incubated overnight at $4{ }^{\circ} \mathrm{C}$ with primary antibodies as shown in Table 2. After washings with T-TBS solution [20 mM Tris/ $\mathrm{HCl}, 137 \mathrm{mM} \mathrm{NaCl}, 0,05 \%$ Tween-20 (pH 7.6)], membranes were incubated with HRP-conjugated secondary antibody for $1 \mathrm{~h}$ at RT and signals were detected using an enhanced chemiluminescence (ECL, Amersham Bioscience, UK) for autoradiography. Band intensity was quantified by computer-assisted image analysis (Adobe Photoshop 6.0, Adobe System Incorporation, USA) calculating pixel number of signal per square centimeter.

\subsection{Reverse transcription-PCR}

The RNA was isolated from the same region of frozen heart biopsy used for protein preparation and it was purified

Table 2

Concentrations of primary and secondary antibodies used for Western blot analysis

\begin{tabular}{lll}
\hline Primary antibodies & Dilution & Secondary antibody diluition \\
\hline Nestin & $1: 250$ & $1: 3000$ \\
C-Kit & $1: 500$ & $1: 12000$ \\
GATA-4 & $1: 500$ & $1: 10000$ \\
\hline
\end{tabular}

according to the protocol recommended by the "quickprep total RNA extraction kit" (Amersham Bioscience) and the RT-PCR was carried out through the "ready-to-go RT-PCR beads" (Amersham Biosciences). The primers used for the amplification of nanog, taube nuss and CD9 genes, respectively, were: 5'-AAGTACCTCAGCCTCCAGCA-3' and $3^{\prime}$ ACCACTGGTTTTTCTGCCAC-5', 5'-AACTTCCAGGACCTGTGTGG-3' and $3^{\prime}$-GTGTGATCGCTCCTGTCTGA5', 5' -TGCAGTGCTTGCTATTGGAC-3' and 3'-GGCGAATATCACCAAGAGGA- $5^{\prime}$. The PCR were performed at $95^{\circ} \mathrm{C}(30 \mathrm{~s}), 58^{\circ} \mathrm{C}(1 \mathrm{~min})$ and $72^{\circ} \mathrm{C}(1 \mathrm{~min})$ for 35 cycles with a Touchgene thermal cycle (Teckgene, USA). A 100 base-pair DNA ladder (Amersham Biosciences, UK) and the products obtained by PCR were visualised on a $2.5 \%$ gel stained with ethidium bromide. The quantitative analysis was performed on 12 heart biopsies and mRNA levels for each gene were evaluated by computer-assisted image analysis (Adobe Photoshop 6.0, Adobe System Incorporation) calculating pixel number of signal per square centimeter.

\subsection{Statistical analysis}

Data are expressed as mean \pm S.D. One way analysis of variance (ANOVA) with Bonferroni multiple comparison test was used to analyse significant differences. The percentage of variability within group was examined by coefficient of variation. Values were considered significantly different at $p<0.05$.

\section{Results}

\subsection{Evaluation of the expression of pericentrin, nestin, c-Kit, GATA-4 and CD34}

In order to examine the presence of dividing mature cardiomyocytes in adult rat heart, we analyzed the expression of pericentrin, that is present in mitosis phase and acts as scaffold protein in recruiting regulatory molecules to the centrosome (Doxsey et al., 1994). As shown in Fig. 1, we did not find any positivity to pericentrin into cardiomyocytes, while it was present in endothelial cells and the number of positive elements was always low (Table 3).

The anti-nestin and anti-GATA-4 antibodies were used to identify cells with myogenic-committed phenotype. Nestin

Table 3

Semi-quantitative analysis of examined protein expression in adult rat heart

\begin{tabular}{llll}
\hline Antibodies & Myocardiocytes & Vessels & Interstitium \\
\hline Pericentrin & - & + & - \\
Nestin & - & + & + \\
GATA-4 & + & - & + \\
CD-34 & - & +++ & - \\
c-Kit & - & + & + \\
Connexin 43 & +++ & - & - \\
\hline
\end{tabular}

Positivity: (+) <33\%; (++) 33-66\%; (+++) >66\%. 

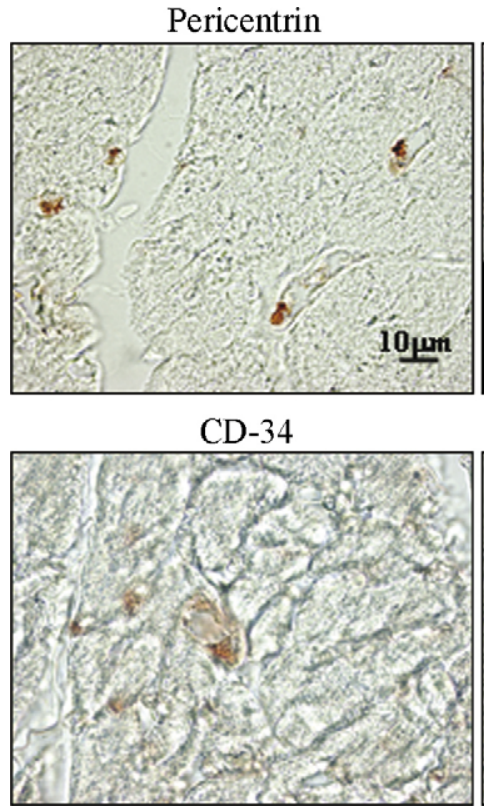

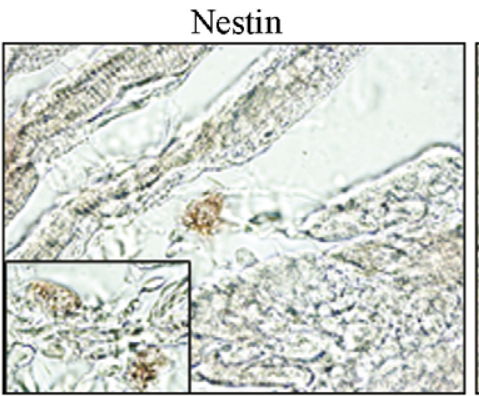

c-Kit

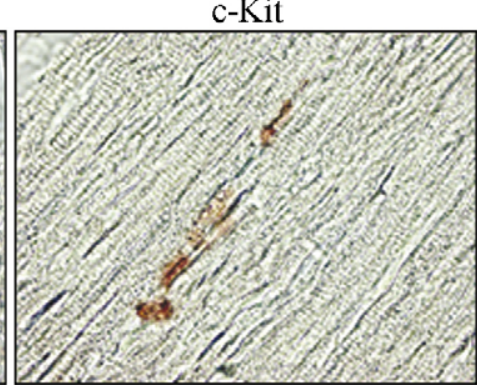

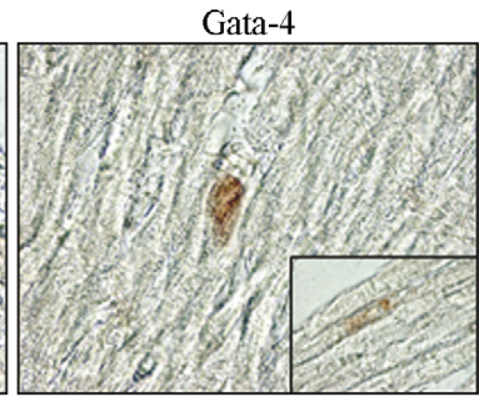

Connexin-43

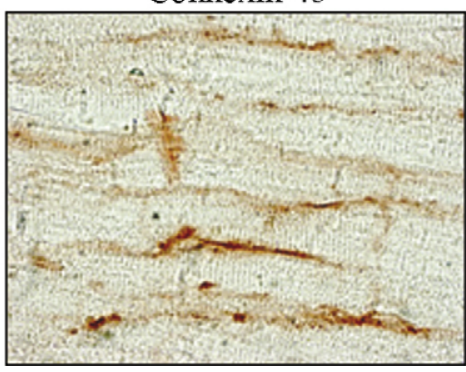

Fig. 1. Representative photomicrographs show the staining of cardiac muscle sections from 24-month-old rats with stem cell-specific antibodies to detect the localization and expression of nestin, GATA-4, CD-34 and c-Kit. Pericentrin expression was examined to find dividing cardiomyocytes. Anti-connexin 43 was used as positive control. Immunohistochemistry results are representative of three independent experiments for each rat heart.

was expressed above all in the cytoplasm of few interstitial cells of myocardium (+), even if a limited number of endothelial cells resulted positive $(+)$ to this marker. GATA-4 was localized in the nuclear and perinuclear region of interstitial and myocardial cells (+).

In addition, CD34 and c-Kit expression was evaluated to examine stem cells committed to one or more specific tissue lineages. CD34 showed a strong positivity (+++) in the endothelial cells of capillary vessels, as well as of endocardium. C-Kit was positive in a low number of interstitial $(+)$ and vascular (+) elements. The expression of nestin,
GATA-4 and c-Kit was mainly localized in subepicardial and subendocardial regions. Connexin- 43 positivity was present in the cardiomyocyte membrane and the positivity was diffuse (+++), as expected. Western blot analyses of Nestin, cKit and GATA- 4 confirmed the expression of these markers in cardiac tissue extracts (Fig. 2A). In particular, quantitative analyses (Fig. 2B) of Western blot results showed that the expression of c-Kit was higher, respectively, by $91.7 \%$ $(p<0.001)$ and $43.8 \%(p<0.01)$ than nestin and GATA-4. No significant difference $(p>0.05)$ was observed between nestin and GATA-4 expression. In addition, the coefficent of
(A)

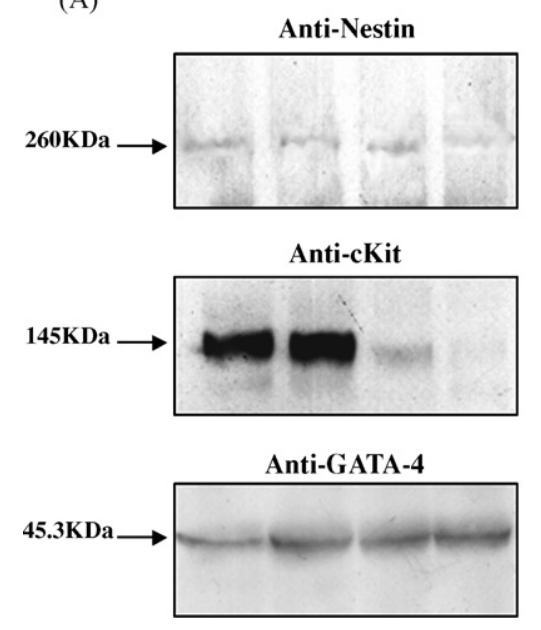

(B)

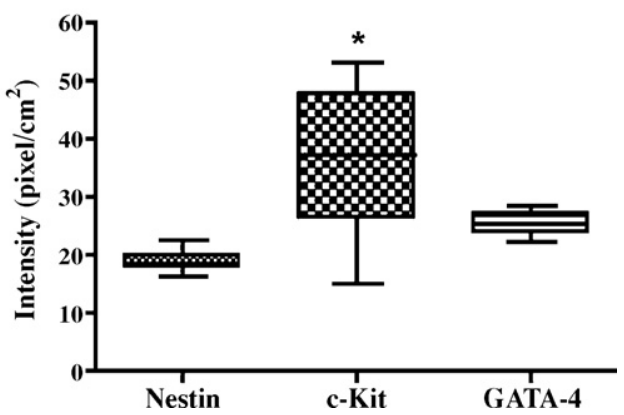

Fig. 2. (A) Heart extracts $(50 \mu \mathrm{g})$ from 12 adult rats were analyzed by immunoblotting to examine the expression of nestin, c-Kit and GATA-4. Figure shows the expression of these markers on four representative samples and confirms the immunoistochemistry results. (B) Protein levels were evaluated by computerassisted image analysis and significant differences were analyzed by ANOVA test. Moreover, differences in the expression of each marker into all examined hearts was assessed by coefficient of variation. The expression and variation coefficient of c-Kit was higher than nestin and GATA-4 ( $\left.{ }^{*} p<0.05\right)$. 
(A)

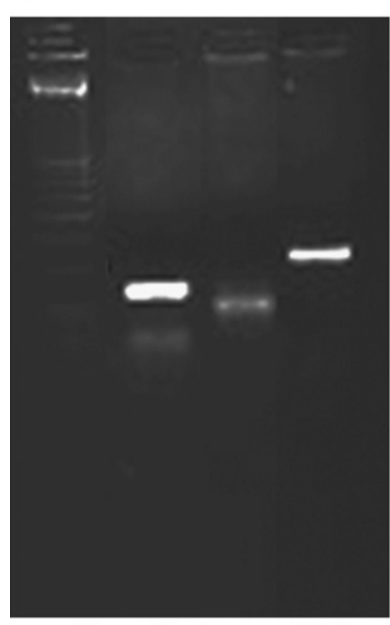

Nanog Taube nuss CD9
(B)

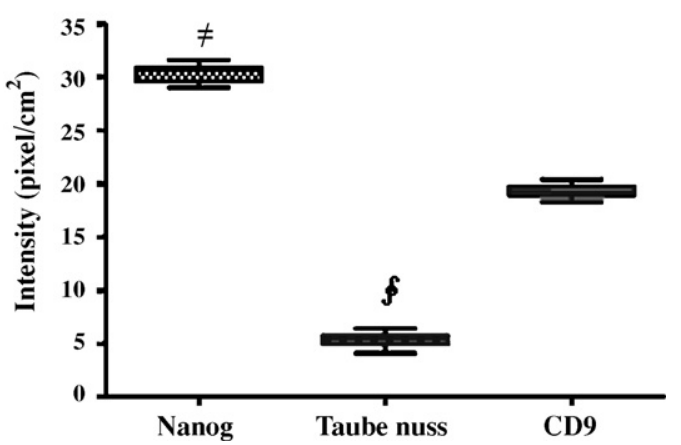

Fig. 3. (A) RT-PCR was performed on RNA extracts from 12 adult rat hearts to study the expression of embryonic stem cell markers. The molecular weight of PCR products corresponds to nanog, taube nuss and CD9 mRNAs, respectively. (B) Band intensity was measured by computer-assisted image analysis and significant differences were examined by ANOVA test. The coefficient of variation was calculated to evaluate the expression difference of each marker into all examined hearts. Nanog mRNA levels were higher than taube nuss and CD9 $(\neq \oint p<0.05)$. The expression of each marker slightly differed in the examined 12 hearts.

variation of c-Kit expression into all examined rat hearts was higher when compared to nestin and GATA-4 (c-Kit, 36.5; nestin, 9.85; GATA-4, 8.07).

\subsection{Assessment of gene expression of nanog, taube nuss and $C D 9$}

In order to detect the presence of stem cells with lineageuncommitted phenotype in adult rat heart, we analyzed the expression of some markers expressed during embryonic development such as nanog, taube nuss and CD9. As antibodies against $\mathrm{CD} 9$, taube nuss and nanog did not provide any immunoreactivity, RT-PCR experiments were used to detect the gene expression. We obtained three gene products of 124, 101 and 220 base pairs corresponding to nanog, taube nuss and CD9 mRNAs, respectively (Fig. 3A). As shown in Fig. 3B, there is a significant difference $(p<0.001)$ in mRNA levels between the examined genes. In particular, nanog mRNA levels were higher than taube nuss and CD9 (30.32 pixel $/ \mathrm{cm}^{2}$ versus 5.36 and $19.32 \mathrm{pixel} / \mathrm{cm}^{2}$ ). Moreover, differently from the nanog and CD9 expression that was similar in the 12 examined hearts (2.94 and 3.27, respectively, of coefficient of variation), the variation of taube nuss levels was higher (10.71 of coefficient of variation).

\section{Discussion}

Recent studies on postinfarcted human hearts have provided the evidence of existance of dividing cardiomyocytes in the peri-infarcted area (Beltrami et al., 2001). Cardiomyocytes in mitosis have also been observed in healthy hearts suggesting that regeneration and death of cardiac muscle cells contribute to the homeostasis of the nondiseased hearts (Anversa and Kajstura, 1998). The identification of cardiomyocyte replication in adult myocardium has stimulated an important debate on the origin of these diving myocytes. It is still unclear whether they are derived from a population of mature cardiomyocytes that have retained their ability to re-enter the cell cycle, from resident cardiac stem cells or from circulating stem cells that have migrated into the heart (Raeburn et al., 2002). Nevertheless, none of these explanations is mutually exclusive and they could all contribute to the cardiac remodelling. In the present study, the absence of pericentrin expression in adult rat cardiomyocytes suggests that the former are not able to divide and they are terminally differentiated cells. Indeed, adult ventricular myocytes are refractory to cell cycle reentry for reasons that include the lack of telomerase activity (Oh et al., 2001). The discovery that adult cardiomyocytes may undergo apoptosis in physiologic conditions (Kang and Izumo, 2000) has proposed the existence of stem cells involved in the physiologic turnover of cardiac muscle tissue.

$\mathrm{Lin}^{-}$and c-Kit ${ }^{+}$cells were recently isolated from rat adult hearts and they showed the ability to replicate and differentiate in cardiomyocytes, endothelial and smooth muscle cells, promoting myocardial repair (Beltrami et al., 2003). In a more recent study, undifferentiated cells from very small fragments of human myocardium were resulted clonogenic and they expressed stem (CD34, c-Kit and Sca-1) and endothelial (flk-1 and CD-31) progenitor cell markers. Moreover, they appeared to have properties of adult cardiac stem cells, able of long-term self-renewal and they could differentiate towards the main specialized cell types of the heart (Messina 
et al., 2004). Other researchers have isolated from adult myocardium c-Kit ${ }^{-}$e Sca- ${ }^{+}$cardiac progenitor cells that expressed a number of cardiogenic transcription factors but not cardiac structural genes (Oh et al., 2003). These cells were induced to differentiate in vitro towards the cardiac myogenic lineage in response to $5^{\prime}$-azacytidine. When administrated intravenously after ischemia/reperfusion experiments, these cells targeted injured myocardium and differentiated into cardiomyocytes, with and without fusion with the host cells (Oh et al., 2003). In addition, resident stem cells positive to nestin, that has been reported to be expressed during embryonic development of the brain and myoblasts (Kachinsky et al., 1995), have been isolated from normal and damaged rat hearts (El-Helou et al., 2005). All these papers suggest the existence of a heterogeneous pool of stem cells in adult myocardium that has not been still completely characterized.

In the present study, the expression of c-Kit, nestin and GATA-4 suggests the existence of committed stem cells in interstitium and near to the vessels of subendocardial and subepicardial region of adult rat heart. A higher expression of c-Kit in comparison with nestin and GATA-4 could indicate a more elevated number of stem cells committed to one or more specific tissue lineages in comparison to the quantity of undifferentiated cells with myogenic-committed phenotype. This discrepancy in expression levels suggests that nestin ${ }^{+}$ and GATA $-4^{+}$cells could be derived from other cell lineages, including c-Kit ${ }^{-}$cells. In this respect, Tomita et al. (2005) showed that neural crest-derived cells migrate and lay in a dormant, undifferentiated state in the heart expressing nestin and GATA-4 or can differentiate into cardiomyocytes or in vitro-derived cardiospheres.

We also postulate that cardiomyogenic potential is different among the various subjects as shown by the different expression of c-Kit in all examined rat hearts. In addition, the expression of specific markers for pluripotent stem cells with lineage-uncommitted phenotype (CD9, taube nuss and nanog) suggests the presence of stem cells from earliest stages of embryogenesis in rat adult heart. Therefore, the presence of embryonic stem cells in adult organ could explain the ability of these cells cultured in vitro to arise cellular elements of different tissue lineages. The different expression of CD9, nanog and taube nuss that we observed in adult myocardium could be associated to the degree of differentiation of embryonic stem cells. Indeed, it has been reported that nanog and CD9 expression was elevated in undifferentiated embryonic stem cells and down-regulated during their differentiation, in concomitance with the loss of the pluripotency (Cui et al., 2004; Hart et al., 2004). Since it has been already demonstrated that the absence of taube nuss causes apoptosis of stem cells from blastocisty inner cellular mass (Voss et al., 2000), we postulate that this factor could be important for stem cell survival even in adult heart.

All these data propose that the mechanisms of cardiac remodelling may occur in vivo, although in our opinion they could not be successful when the necrotic area is too large, especially in late age. A possible explanation may be the reduction of regenerative potential correlated with the aging of the organism. Embryonic stem cells could be more abundant in the heart during development (at birth) and decrease with progression to an adult phenotype. In adult myocardium, stem cells are probably spatially segregated in niches that hide them from exposure to inductive signals of differentiation (Watt and Hogan, 2000). In agreement Behfar et al. (2002) have shown that embryonic stem cells transplanted into mouse hearts differentiated into cardiomyocytes only when the cardiac paracrine pathway mediated by TGF- $\beta$ and BMP2 was intact. Therefore, the study of signal molecules inducing differentiation of stem cells versus the cardiac lineage could be determinant to enhance the efficiency of the regenerative potential of these cells.

Recently it has been shown that TGF-beta induced the myogenic differentiation of $\mathrm{c}-\mathrm{Kit}^{+}$stem cells by upregulation of GATA-4 and NKx-2.5 expression in an acute myocardial infarction model. In particular, histologic analyses revealed newly regenerated myocardium in the left ventricular anterior wall after the implantation of TGF-beta-preprogrammed cells in comparison with untreated cells (Li et al., 2005). It is our opinion that the study of myogenic potential could be important to identify markers specific of stem cells in in vivo adult myocardium that may be used to purify these cells and evaluate their regenerative ability.

\section{Acknowledgments}

We are grateful to Dr. Marianna Zummo for the English revision of the manuscript. This work was supported by a grant from Fondi MURST ex-60\%.

\section{References}

Anversa, P., Kajstura, J., 1998. Ventricular myocytes are not terminally differentiated in the adult mammalian heart. Circ. Res. 83, 1-14.

Bhattacharya, B., Miura, T., Brandenberger, R., Mejido, J., Luo, Y., Yang, A.X., Joshi, B.H., Ginis, I., Thies, R.S., Amit, M., Lyons, I., et al., 2004. Gene expression in human embryonic stem cells: unique molecular signature. Blood 103, 2956-2964.

Beltrami, A.P., Urbanek, K., Kajstura, J., Yan, S.M., Finato, N., Bussani, R., Nadal-Ginard, B., Silvestri, F., Leri, A., Beltrami, C.A., Anversa, P., 2001. Evidence that human cardiac myocytes divide after myocardial infarction. New Engl. J. Med. 344, 1750-1757.

Behfar, A., Zingman, L.V., Hodgson, D.M., Rauzier, J.M., Kane, G.C., Terzic, A., Pucèat, M., 2002. Stem cell differentiation requires a paracrine pathway in the heart. FASEB J. 16, 1558-1564.

Beltrami, A.P., Barlucchi, L., Torella, D., Baker, M., Limana, F., Chimenti, S., Kasahara, H., Rota, M., Musso, E., Urbaneck, K., Leri, A., Kajstura, J., Nadal-Ginard, B., Anversa, P., 2003. Adult cardiac stem cells are multipotent and support myocardial regeneration. Cell 114, 763-776.

Chambers, I., Colby, D., Robertson, M., Nichols, J., Lee, S., Tweedie, S., Smith, A., 2003. Functional expression cloning of nanog, a pluripotency sustaining factor in embryonic stem cells. Cell 113, 643-655.

Cui, L., Johkura, K., Yue, F., Ogiwara, N., Okouchi, Y., Asanuma, K., Sasaki, K., 2004. Spatial distribution and initial changes of SSEA-1 and other cell adhesion-related molecules on mouse embryonic stem cells before and during differentiation. J. Histochem. Cytochem. 52, 1447-1457. 
Doxsey, S.J., Stein, P., Evans, L., Calarco, P.D., Kirschner, M., 1994. Pericentrin, a highly conserved centrosome protein involved in microtubule organization. Cell 76, 639-650.

El-Helou, V., Dupuis, J., Proulx, C., Drapeau, J., Clement, R., Gosselin, H., Villeneuve, L., Manganas, L., Calderone, A., 2005. Resident nestin ${ }^{+}$ neural-like cells and fibers are detected in normal and damaged rat myocardium. Hypertension 46, 1219-1225.

Evans, M.J., Kaufman, M.H., 1981. Establishment in culture of pluripotential cells from mouse embryos. Nature 292, 154-156.

Giuliano, C.J., Kerley-Hamilton, J.S., Bee, T., Freemantle, S.J., Manickaratnam, R., Dmitrovsky, E., Spinella, M.J., 2005. Retinoic acid represses a cassette of candidate pluripotency chromosome $12 \mathrm{p}$ genes during induced loss of human embryonal carcinoma tumorigenicity. Biochim. Biophys. Acta 1731, 48-56.

Hart, A., Hartley, L., Ibrahim, M., Robb, L., 2004. Identification, cloning and expression analysis of the pluripotency promoting nanog genes in mouse and human. Dev. Dyn. 230, 187-198.

Kachinsky, A.M., Dominov, J.A., Miller, J.B., 1995. Intermediate filaments in cardiac myogenesis: nestin in the developing mouse heart. J. Histochem. Cytochem. 43 (8), 843-847.

Kang, P.M., Izumo, S., 2000. Apoptosis and heart failure: a critical review of the literature. Circ. Res. 86, 1107-1113.

Li, T.S., Hayashi, M., Ito, H., Furutani, A., Murata, T., Matsuzaki, M., Hamano, K., 2005. Regeneration of infarcted myocardium by intramyocardial implantation of ex vivo transforming growth factor-betapreprogrammed bone marrow stem cells. Circulation 111, 2438-2445.

Malouf, N.N., Coleman, W.B., Grisham, J.W., Lininger, R.A., Madden, V.J., Sproul, M., Anderson, P.A.W., 2001. Adult-derived stem cells from the liver become myocytes in the heart in vivo. Am. J. Pathol. 158, 1929-1935.

Messina, E., De Angelis, L., Frati, G., Morrone, S., Cimenti, S., Fiordaliso, F., Salio, M., Battaglia, M., Latronico, M.V.G., Coletta, M., Vivarelli, E., Frati, L., Cossu, G., Giacomello, A., 2004. Isolation and expansion of adult cardiac stem cells from human and murine heart. Circ. Res. 95, 911-921.

Oh, H., Taffet, G.E., Youker, K.A., Entman, M.L., Overbeek, P.A., Michael, L.H., Schneider, M.D., 2001. Telomerase reverse transcriptase promotes cardiac muscle cell proliferation, hypertrophy, and survival. Proc. Natl. Acad. Sci. U.S.A. 98, 10308-10313.

Oh, H., Bradfute, S.B., Gallardo, T.D., Nakamura, T., Gaussin, V., Mishina, Y., Pocius, J., Michael, L.H., Behringer, R.R., Garry, D.J., Entman, M.L.,
Schneider, M.D., 2003. Cardiac progenitor cells from adult myocardium: homing, differentiation, and fusion after infarction. Proc. Natl. Acad. Sci. U.S.A. 100, 12313-12318.

Oka, M., Tagoku, K., Russel, T.L., Nakano, Y., Hamazaki, T., Meyer, E.M., Yokota, T., Terada, N., 2002. CD9 is associated with leukemia inhibitory factor-mediated maintenance of embryonic stem cells. Mol. Biol. Cell $13,1274-1281$

Orkin, S.H., Zon, L.I., 2002. Hematopoiesis and stem cells: plasticity versus development heterogeneity. Nat. Immunol. 3, 323-328.

Orlic, D., Kajstura, J., Chimenti, S., Limana, F., Jakoniuk, I., Quaini, F., Nadal-Ginard, B., Bodine, D.M., Leri, A., Anversa, P., 2001. Mobilized bone marrow cells repair the infarcted heart, improving function and survival. PNAS 98, 10344-10349.

Raeburn, C.D., Zimmerman, M.A., Arya, J., Banerjee, A., Harken, A.H., 2002. Stem cells and myocardial repair. J. Am. Coll. Surg. 195, 686-693.

Shintani, S., Murohara, T., Ikeda, H., Ueno, T., Honma, T., Katoh, A., Sasaki, K., Shimada, T., Oike, Y., Imaizumi, T., 2001. Mobilization of endothelial progenitor cells in patients with acute myocardial infarction. Circulation 103, 2776-2779.

Toma, C., Pittenger, M.F., Cahill, K.S., Byrne, B.J., Kessler, P.D., 2002. Human mesenchymal stem cells differentiate to a cardiomyocyte phenotype in the adult murine heart. Circulation 105, 9398.

Tomita, S., Li, R.K., Weisel, R.D., Mickle, D.A., Kim, E.J., Sakai, T., Jia, Z.Q., 1999. Autologous transplantation of bone marrow cells improves damaged heart function. Circulation 100 (Suppl. II), 1124711256.

Tomita, Y., Matsumura, K., Wakamatsu, Y., Matsuzaki, Y., Shibuya, I., Kawaguchi, H., Ieda, M., Kanakubo, S., Shimazaki, T., Ogawa, S., Osumi, N., Okano, H., Fukuda, K., 2005. Cardiac neural crest cells contribute to the dormant multipotent stem cell in the mammalian heart. J. Cell Biol. 170, 1135-1146.

Voss, A.K., Thomas, T., Petrou, P., Anastassiadis, K., Sholer, H., 2000. Taube nuss is a novel gene essential for the survival of pluripotent cells of early mouse embryos. Development 127, 5449-5461.

Watt, F.M., Hogan, B.L., 2000. Out of Eden: stem cells and their niches. Science 287, 1427-1430.

Yoder, M.C. 2004. Blood cell progenitors: insights into the properties of stem cells. The anatomical record part A 276A: 66-74.

Young, H.E., Black, A.C., Jr 2004. Adult stem cells. The anatomical record part A 276A: 75-102. 